\title{
Estudo da Dissolução de Polieletrólitos em Água Pura e em Meios de Força lônica Moderada
}

\author{
Ricardo C. Michel, Cristina T. Andrade e Wayne F. Reed
}

Resumo: O processo de dissolução de polieletrólitos em pó foi monitorado pela passagem da amostra através de um filtro em linha, um detector de espalhamento de luz multi-ângulo, um refratômetro e um viscosímetro capilar. Quando os polieletrólitos foram dissolvidos em soluções de força iônica moderada, o seu comportamento de dissolução foi similar ao de polímeros neutros. No entanto, quando dissolvidos em água pura, uma pequena população de agregados surgiu no início do processo de dissolução. A conformação dos agregados evoluiu desde uma distribuição gaussiana esféricamente simétrica de densidade de massa até estruturas similares a novelos aleatórios, contendo diversas cadeias. Ao final do processo de dissolução, as cadeias de polieletrólito se encontravam rígidas, distendidas e bem dispersas.

Palavras-chave: Dissolução, polieletrólitos, espalhamento de luz, agregados, não-equilíbrio.

Introdução

A dissolução de polímeros é um processo de considerável importância tecnológica, com aplicação em áreas tão diversas quanto eletrônica, farmácia, fotografia e alimentação. No entanto, não existem métodos estabelecidos ou largamente aceitos para acompanhar a dissolução de um dado polímero em um dado solvente $e^{[1,2]}$.

Este trabalho teve por objetivo acompanhar o processo de dissolução de polímeros hidrossolúveis polieletrólitos em água pura e em meios de força iônica moderada através da aplicação de técnicas de espalhamento de luz estático em função do tempo (TDSLS), refratometria (RI) e viscosimetria (SC), tendo sido esclarecidas algumas questões referentes à natureza de não-equilíbrio da pequena população de agregados presentes nas soluções de força iônica reduzida.

O processo de dissolução pode ser descrito da seguinte forma ${ }^{[3,4,5]}$ : um grão esférico de material polimérico de raio inicial $R_{o}$ e densidade $\rho_{0}$, em presença de solvente, após um período inicial de umedecimento, começa a liberar cadeias de polímero na solução, com um fluxo de massa $J$ (dado em $\mathrm{g} / \mathrm{cm}^{2} \mathrm{~s}$ ). Assumindo-se um valor de $\mathrm{J}$ constante e que o tempo necessário para o umedecimento e para a formação da camada de gel em torno dos grãos de material sêco é negligenciável frente ao tempo necessário para a dissolução dos grãos, tem-se que a concentração de polímero dissolvido, como função do tempo, $\mathrm{Cp}(\mathrm{t})$, é: com

$$
\mathrm{C}_{\mathrm{p}}(\mathrm{t})=\mathrm{C}_{\mathrm{o}}\left[1-\left(1-\mathrm{kt} / \mathrm{R}_{\mathrm{o}}\right)^{3}\right], \quad \mathrm{kt} \leq \mathrm{R}_{\mathrm{o}}
$$

$$
\mathrm{C}_{\mathrm{p}}(\mathrm{t})=\mathrm{C}_{\mathrm{o}} \text { para } \mathrm{kt}>\mathrm{R}_{\mathrm{o}}
$$


aonde $\mathrm{C}_{\mathrm{o}}$ é a concentração inicial em massa dos grãos na solução e $\mathrm{k}=\mathrm{J} / \mathrm{r}_{\mathrm{o}}$.

A velocidade inicial normalizada da dissolução é dada por:

$$
\mathrm{V}_{\text {in }}=\left.\frac{1}{\mathrm{C}_{0}} \frac{\mathrm{dC}_{\mathrm{p}}(\mathrm{t})}{\mathrm{dt}}\right|_{\mathrm{t}=0}=\frac{3 \mathrm{k}}{\mathrm{R}_{0}}
$$

A inclinação inicial da curva ' $C_{p}(t)$ versus $t$ ' define a curva completamente, uma vez que $k / R_{o}$ é o único parâmetro. Qualquer propriedade física proporcional à concentração de material dissolvido pode ser utilizada para se acompanhar $\mathrm{C}_{\mathrm{p}}(\mathrm{t})$ ao longo do tempo. Nos experimentos realizados neste trabalho utilizou-se um detector de índice de refração diferencial com célula de fluxo para obterse a medida de concentração diretamente.

\section{Experimental}

Uma bomba peristáltica foi usada para remover a amostra sob agitação de um béquer de vidro de $250 \mathrm{ml}$ e bombeá-la através de um filtro de membrana de ésteres de celulose conectado em série com um detector de espalhamento de luz multiângulo Wyatt Dawn-F, um detector de índice de refração ERMA ERC-7522 e um viscosímetro capilar montado no laboratório em torno de um transdutor de diferencial de pressão Valydine DP15-28. Foram utilizados filtros Millipore com tamanhos de poro de $0.45 \mu \mathrm{m}$, $0.22 \mu \mathrm{m}$ e $0.10 \mu \mathrm{m}$.

Em cada experimento bombeou-se o solvente puro em circuito fechado através do sistema por cerca de 15 minutos, para aquisição das linhas de base dos detectores. Logo após, adicionou-se $15 \mathrm{mg}$ de polímero em pó aos $100 \mathrm{ml}$ de solvente, adquirindo-se os dados dos detectores a intervalos de 5 segundos. O polímero principal utilizado foi o poli(estireno sulfonato de sódio) (NaPSS), com $\mathrm{M}_{\mathrm{w}}=46000 \mathrm{~g} / \mathrm{mol}$ e $\mathrm{M}_{\mathrm{w}} / \mathrm{M}_{\mathrm{n}}=1.10$. Foram utilizados também pectinas, ácido hialurônico e hidrobrometo de poli-lisina (PLL), obtendo-se o mesmo tipo de comportamento, além de dextranas e poli(vinil pirrolidona) como material não-polieletrólito de controle. Como solventes utilizou-se água pura e solução aquosa de $\mathrm{NH}_{4} \mathrm{NO}_{3} 0,1 \mathrm{M}$, referida daqui por diante como 'solução salina'.

\section{Resultados e Discussão}

\section{Velocidade de Dissolução}

A Figura 1, apresenta as curvas de dissolução para NaPSS em água pura e em solução salina.

A Figura 1 mostra que as velocidades de dissolução para as amostras diluídas tanto em água pura, com qualquer dos filtros utilizados, quanto em solução salina, com filtro de $0.45 \mathrm{~mm}$, têm essencialmente o mesmo valor. Utilizando-se os valores obtidos de $\mathrm{V}_{\text {in }}$ de $0.0188 \mathrm{~s}^{-1}$ e $0.0181 \mathrm{~s}^{-1}$ para o NaPSS em água pura e em solução salina, respectivamente, obteve-se uma excelente concordância entre os dados experimentais e a curva teórica ajustada pela equação 1 . Desta forma, a dependência cúbica com o tempo sugerida na equação 1, com fluxo J constante, descreve adequadamente o processo de dissolução observado.

$\mathrm{O}$ fato de que $\mathrm{V}_{\text {in }}$ não depende da força iônica é surpreendente, uma vez que se poderia supor que a repulsão eletrostática entre as cadeias no caso da água pura poderia fazer com que as cadeias se liberassem do grão hidratado mais rapidamente. $\mathrm{O}$ fato de que a força iônica não tem efeito sobre a velocidade inicial sugere que a etapa limitadora da velocidade de dissolução depende de processos dentro da camada de gel, aonde existe eletroneutralidade, devido à presença dos contraíons do polieletrólito, presentes mesmo no caso da água pura.

A figura 1 também mostra os dados obtidos para NaPSS filtrado através de filtros com $0.1 \mu \mathrm{m}$ e $0.22 \mu \mathrm{m}$ de diâmetro de poro. Assim, pode-se

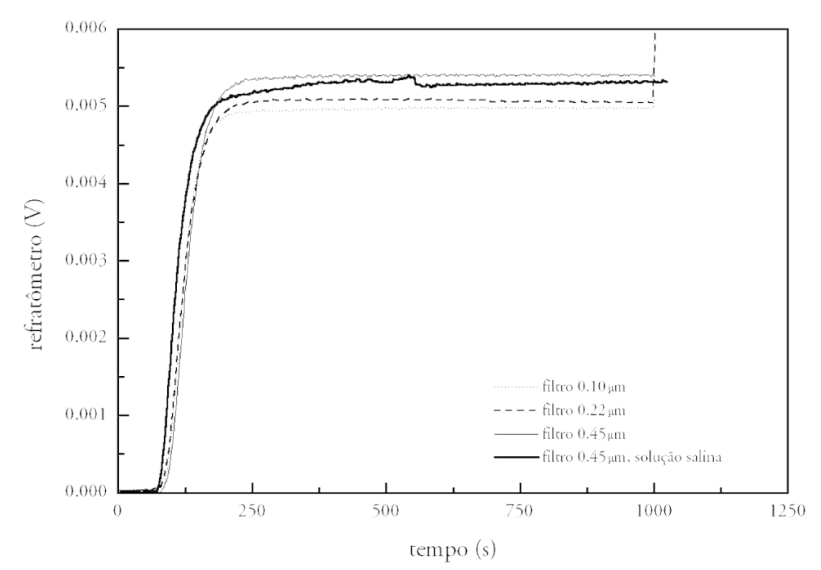

Figura 1a. Voltagem em RI versus tempo para NaPSS $0.15 \mathrm{mg} / \mathrm{ml}$ em água pura sob diferentes filtrações e em solução salina com filtração em filtro de $0.45 \mu \mathrm{m}$. 
utilizar a figura 1 para estimar, em cada situação, a quantidade de material presente na forma de agregados. Assumindo-se que uma quantidade desprezível de material está presente na forma de agregados no caso de filtração em $0.1 \mathrm{~mm}$, isto é, que a maioria dos agregados foi bloqueada pelo filtro e não foi desfeita por cisalhamento, então o nível dos patamares permite estimar que a fração do material na forma de agregados é de $0.067 \mathrm{e}$ 0.050 para os filtros $0.45 \mu \mathrm{m}$ e $0.22 \mu \mathrm{m}$, respectivamente. Ou seja, apenas uma pequena parte do material está presente na forma de agregados na solução filtrada. Usando-se as estimativas da quantidade de agregados, obtidas acima, tem-se que o valor de $\mathrm{M}_{\mathrm{w}}$ para os dados de filtração a $0.45 \mu \mathrm{m}$ devem ser multiplicados por cerca de 15 vezes, enquanto os dados para a filtração por $0.22 \mu \mathrm{m}$ devem ser multiplicados por aproximadamente 20. Uma vez que a concentração de agregados é muito pequena e porque se espera que os agregados apresentem um $\mathrm{A}_{2}$ relativamente pequeno, não deve existir quase nenhuma supressão de espalhamento.

\section{Espalhamento de Luz e a Formação Inicial de Agregados}

A Figura 2 apresenta os dados de espalhamento de luz em função do tempo (TDSLS) para os mesmos experimentos apresentados na figura 1 .

Em contraste com o comportamento mostrado por RI, o comportamento de TDSLS para a dissolução de NaPSS tem forte dependência do uso de água pura ou de solução salina.

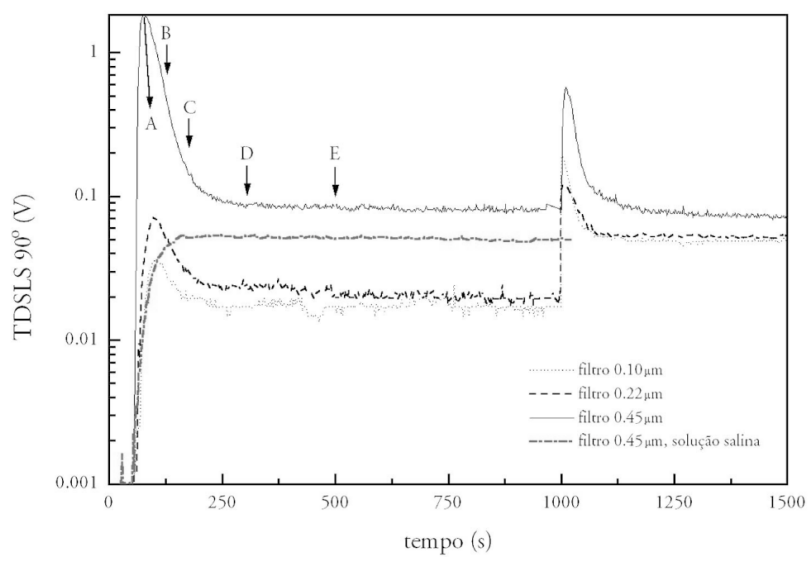

Figura 2. Voltagem em TDSLS versus tempo para os mesmos experimentos mostrados na figura 1 . Os pontos de 'A' até ' $E$ ' servem como referência para a análise dos dados.
No caso da água pura, o sinal de TDSLS produz um pico intenso, aproximadamente no mesmo instante em que o primeiro aumento de RI ocorre, e então começa a decrescer até atingir um patamar, aproximadamente no tempo que o RI necessita para atingir o seu próprio patamar. Este comportamento de TDSLS foi observado também para outros polieletrólitos, incluindo PLL, NaPSS de grande massa molecular e pectina. Para dissoluções em solução salina a curva de TDSLS alcança o seu patamar monotonicamente, da mesma forma que ocorre com o RI e, de fato, se sobrepõe à curva de RI, apresentando a mesma inclinação reduzida ('inclinação reduzida' é a inclinação inicial dividida pelo nível do patamar da curva). É esperado que ocorra esta sobreposição se $M_{w}$ se mantém constante ao longo do processo e se os efeitos de $A_{2}$ são pequenos, como o são no caso de solução salina, uma vez que I(q) é proporcional a $\mathrm{cM}_{\mathrm{w}}$. A rapidez do surgimento dos agregados sugere que estes se destacam dos grãos macroscópicos originais.

A 1000 segundos após o início da dissolução, uma pequena quantidade de uma solução aquosa de $\mathrm{NaCl} 550 \mathrm{mM}$ foi adicionada às soluções em água pura da Figura 2, elevando a força iônica para $25 \mathrm{mM}$. Observa-se então a formação de um pico e a alteração do nível de cada patamar de intensidade de luz espalhada. Um efeito que pode ocorrer em função da adição do sal é que agregados maiores do que o tamanho dos poros do filtro, no lado do filtro mais próximo do frasco de dissolução, começam a dissolver e cruzam o filtro, produzindo os pequenos picos presentes na Figura 2 após o ponto de adição do sal.

O nível de espalhamento da curva para o filtro de $0.45 \mu \mathrm{m}$ diminui levemente quando o sal é adicionado, enquanto os níveis das curvas com os filtros $0.1 \mu \mathrm{m}$ e $0.22 \mu \mathrm{m}$ aumentam até atingir o mesmo nível apresentado pelo NaPSS em solução salina.

Nas curvas para os filtros pequenos existe uma população inicial pequena de agregados, a qual rapidamente diminui. O aumento no nível de luz espalhada com a adição de sal é devido à queda em $\mathrm{A}_{2}$ que ocorre em um polieletrólito quando a força iônica é aumentada. O fato de que a intensidade de luz espalhada após a adição de sal é a mesma que para o NaPSS originalmente dissolvido em solução salina indica que não há uma po- 
pulação remanescente de agregados que seja detectável após a adição de sal.

Para a curva da filtração em $0.45 \mu \mathrm{m}$, em contraste, a intensidade de luz espalhada diminui levemente após a adição de sal, indicando que o sal deve estar promovendo a redução da população de agregados, pois se os agregados permanecessem, somando-se o seu espalhamento com o espalhamento aumentado das cadeias individuais (como o visto nas outras curvas), a intensidade total de luz espalhada deveria aumentar. Da mesma forma, uma vez que o nível de espalhamento após a adição de sal não cai para o mesmo patamar que aquele das outras curvas, deduz-se que a dissolução dos agregados não é completa na escala de tempo deste experimento.

Para mostrar que este comportamento não é um artefato da técnica de TDSLS quando água pura é usada, a Figura 3 mostra os dados obtidos para um polímero neutro, dextrana, em água pura e em solução salina.

As curvas obtidas para dextrana em água pura e em solução salina são aproximadamente idênticas. O mesmo tipo de resultados foi também encontrado para poli(vinil pirrolidona), neutra. Assim fica claro que o pico apresentado em TDSLS para o caso da água pura é característico dos polieletrólitos, ao menos destes estudados.

No seu máximo de massa molecular (com os fatores de correção discutidos acima) de $2 \times 10^{9}$ $\mathrm{g} / \mathrm{mol}$, os agregados de NaPSS em água pura, filtrados a $0.45 \mu \mathrm{m}$, compreendem mais de 40000 cadeias individuais de NaPSS de $46000 \mathrm{~g} / \mathrm{mol}$ cada, e a distribuição de massa de um tal agregado é

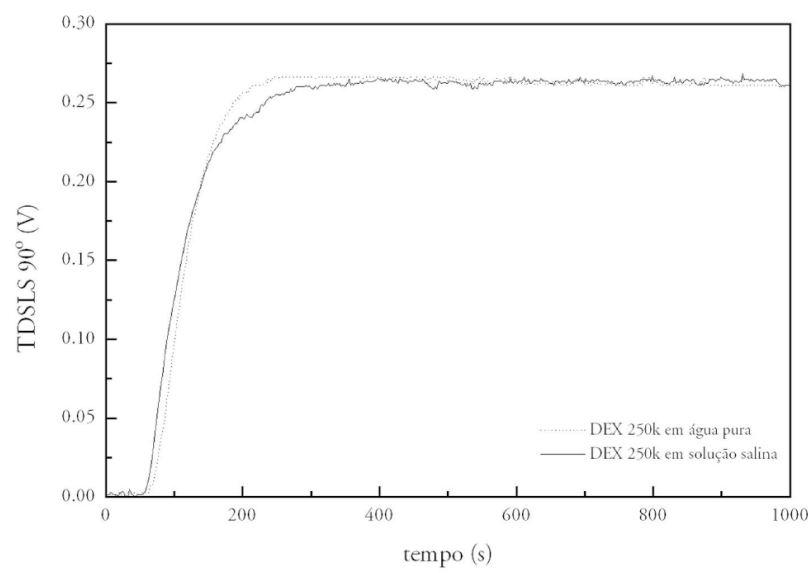

Figura 3. Voltagem em TDSLS versus tempo para um experimento de controle envolvendo dextrana neutra em água pura e solução salina. Nenhum pico surge e a velocidade inicial é a mesma para ambas as amostras. cerca de 17000 vezes mais densa do que aquela de uma cadeia singular bem dispersa (a densidade é computada de $\left.\mathrm{M}_{\mathrm{w}} /\left(4 \pi \mathrm{R}_{\mathrm{g}, \mathrm{z}}{ }^{3 / 3}\right)\right)$. Após este pico tanto a massa quanto a densidade diminuem drasticamente. A densidade dos agregados que persistem após a filtração em $0.22 \mu \mathrm{m}$ é cerca de 1400 vezes maior do que aquela de uma cadeia bem dispersa. Na verdade, estas grandes diferenças na estrutura dos agregados refletem-se na forma de seus envelopes de espalhamento.

\section{Viscosimetria}

O sinal detectado pelo viscosímetro capilar durante a evolução dos experimentos presentes na Figura 1 é apresentado na Figura 4.

Não há na Figura 4 nenhum pico similar a aqueles presentes nas curvas de TDSLS da figura 2, mostrando que a viscosidade relativa não é sensível aos agregados detectados por TDSLS. Isto é mais uma evidência de que apenas uma pequena fração de massa da amostra está presente na forma de agregados.

O elevado sinal de viscosidade em água pura é devido a cadeias bem dispersas, as quais se encontram bastante estendidas, graças à ausência de blindagem iônica, apresentando, portanto, elevada viscosidade intrínseca. O NaPSS em solução salina na Figura 4 apresenta uma viscosidade intrínseca tão reduzida, devido à blindagem iônica, que não se produz um sinal mensurável. Aos 1000 segundos, quando adiciona-se $\mathrm{NaCl}$ às soluções em água pura, suas viscosidades rapidamente se igualam ao valor de viscosidade da solução salina.

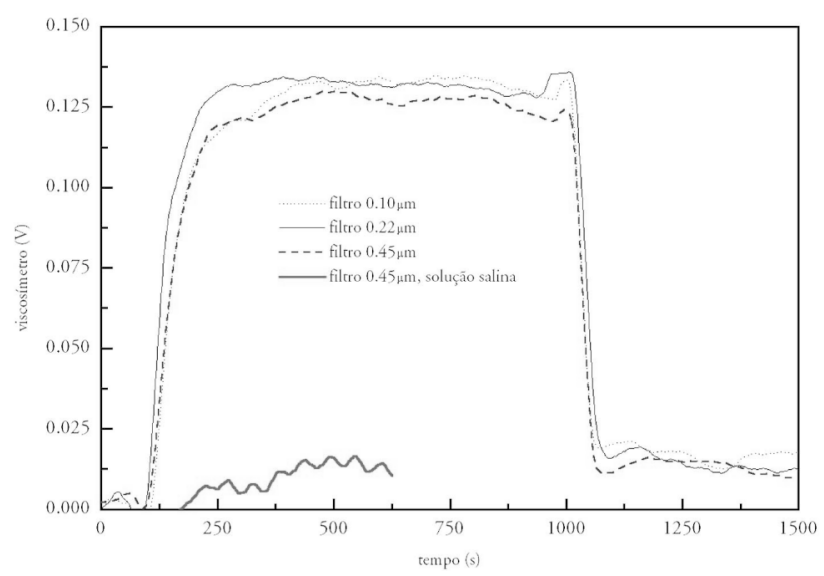

Figura 4. Voltagem do viscosímetro versus tempo para os experimentos da Figura 1. 
A Figura 4 também mostra que todas as curvas são similares (isto é, apresentam a mesma inclinação reduzida), confirmando a hipótese de que a maioria dos grãos de polieletrólitos dissolvemse na mesma velocidade tanto em água pura, quanto em solução salina.

\section{Forma dos Agregados}

A Figura 5 dá a intensidade de luz espalhada em função do ângulo, representada como $\mathrm{Kc} / \mathrm{I}(\mathrm{q})$, para agregados de NaPSS em água pura, filtrados em $0.45 \mu \mathrm{m}$ no ponto 'B' da figura 2 .

A curvatura positiva presente para os pontos desde o início da Figura 2 até próximo do ponto ' $D$ ' sugere fortemente a presença de pontos de espalhamento ('scatterers') esferoidais densos, ao invés de novelos aleatórios.

$O$ fator forma para uma esfera rígida uniforme não ajusta bem os dados obtidos, de forma que os agregados não devem apresentar esta conformação.

No entanto, para uma distribuição de densidade de massa gaussiana esfericamente simétrica:

$$
\rho(r)=\rho_{0} \exp \left[-(r / a)^{2}\right\rfloor
$$

tem-se que o fator forma dentro da aproximação de Rayleigh-Debye é (bo8):

$$
P(q)=\exp \left(-q^{2} a^{2} / 2\right)
$$

e o comprimento característico de decaimento é relacionado com o raio de giração por

$$
\mathrm{R}_{\mathrm{g}}=\sqrt{\frac{3}{2} \mathrm{a}}
$$

Um ajuste desta curva de $\mathrm{P}(\mathrm{q})$ da equação 4 é mostrado sobre os pontos da figura 5. O ajuste é bom e fornece um valor de $\mathrm{R}_{\mathrm{g}, \mathrm{z}}$, pela equação 5 , de $972 \AA$, que está de acordo com o valor obtido por extrapolação linear a ângulo pequeno.

Assim, logo que surgem, os agregados podem ser descritos como sendo esféricamente simétricos, cuja densidade diminui de forma gaussiana desde o centro denso até a sua borda.

A curvatura de $\mathrm{Kc} / \mathrm{I}$ permanece positiva para todos os pontos no lado descendente do pico da Figura 2. Quando o patamar é alcançado a curva $\mathrm{Kc} / \mathrm{I}$ para o ponto ' $\mathrm{D}$ ' não mais possui curvatura

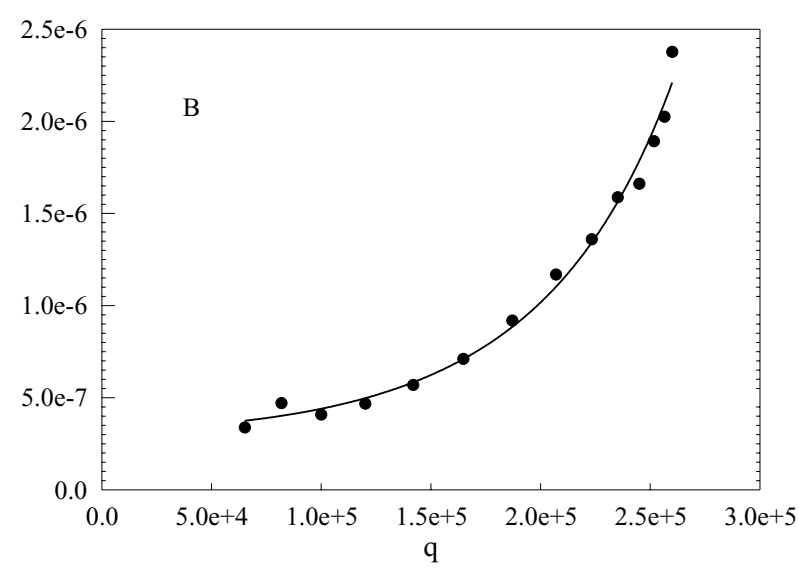

Figura 5. 'Kc/I versus q' para NaPSS em água pura com filtração em $0.45 \mu \mathrm{m}$, no ponto B da Figura 2. A curvatura positiva corresponde a partículas esferoidais densas com distribuição gaussiana de massa (equação 4).

positiva, sugerindo uma estrutura do tipo novelo aleatório.

A grande diminuição da densidade das estruturas é consistente com a evolução dos agregados partindo de esferóides gaussianos até adquirirem estruturas do tipo novelo aleatório. Isto sugere que os agregados, no caso das filtrações a $0.45 \mu \mathrm{m}$, começam como estruturas densas, aumentam de tamanho com a penetração do solvente, perdendo uma considerável quantidade de massa uma vez inchados, e, finalmente, atingindo um estado metaestável.

Em contraste com a filtração a $0.45 \mu \mathrm{m}$, as curvas de $\mathrm{Kc} / \mathrm{I}$ para os agregados que sobrevivem à filtração a $0.22 \mu \mathrm{m}$ nunca apresentam curvatura positiva, não sendo, portanto, identificáveis como estruturas gaussianas esferoidais densas.

\section{Conclusões}

A velocidade de dissolução do NaPSS em água pura e em solução salina é a mesma e segue o modelo simples de dependência cúbica com o tempo, predito para um fluxo constante de cadeias de polieletrólitos deixando o grão macroscópico original.

Os agregados que surgem durante a dissolução em água pura constituem uma fração pequena da massa total do material dissolvido e eventualmente desaparecem. A adição de $\mathrm{NaCl}$ causa a dissolução irreversível dos agregados. Não há traços detectáveis de agregados quando a dissolução ocorre em solução salina de moderada força iônica. A 
diminuição do tamanho de poro do filtro leva à diminuição tanto da quantidade quanto do tamanho inicial dos agregados. Os agregados parecem estar presentes no pó do polieletrólito sêco, sendo diretamente relacionados com a história termodinâmica da produção do material seco.

Os agregados evoluem no tempo desde uma distribuição gaussiana de densidade de massa de simetria esférica até atingirem um estado que se assemelha a novelos aleatórios contendo várias cadeias e, finalmente, dissolvem-se completamente, dando origem a cadeias bem dispersas e rígidas de polieletrólitos.

Uma vez que os agregados são inerentemente instáveis, dissolvendo-se ao longo do tempo, não são viáveis presentemente medidas quantitativas exatas das propriedades dos agregados em termos de tamanho, massa e fração de massa, uma vez que existirão variações significativas de experimento para experimento e, certamente, entre diferentes fontes de material. No entanto, as tendências principais no comportamento destes parâmetros foram apresentadas neste trabalho.
Agradecimento

Os autores agradecem o financiamento da CAPES/PDEE.

\section{Referências Bibliográficas}

1. Reed, W. F. - Ber. Bunsenges. Phys. Chem. 100, 685-695 (1996).

2. Ranade, V. V. \& Mashelkar, R. A. - AIChE Journal vol. 41, No. 3, 666-676 (1995).

3. Tu, Y. \& Ouano, A. C. - IBM J. Res. Dev. 21, 131-142 (1977).

4. Devotta, I.; Ambeskar, V. D.; Mandhare, A. B. \& Mashelkar, R. A. - Chemical Engineering Science vol. 49, No. 5, 645-654 (1994).

5. Brochard, F. \& Gennes, P. G. de - Physico Chemical Hydrodynamics vol. 4, No. 4, 313322 (1983). 